\title{
Stability Analysis and Investigation of a Magnetoelastic Beam Subjected to Axial Compressive Load and Transverse Magnetic Field
}

\author{
Mei-Feng Liu ${ }^{1}$ and Tai-Ping Chang ${ }^{2}$ \\ ${ }^{1}$ Department of Applied Mathematics, I-Shou University, Kaohsiung 840, Taiwan \\ ${ }^{2}$ Department of Construction Engineering, National Kaohsiung First University of Science \& Technology, \\ Kaohsiung 824, Taiwan \\ Correspondence should be addressed to Mei-Feng Liu, meifeng@isu.edu.tw
}

Received 22 December 2009; Revised 22 April 2010; Accepted 3 June 2010

Academic Editor: Carlo Cattani

Copyright $\odot 2010$ M.-F. Liu and T.-P. Chang. This is an open access article distributed under the Creative Commons Attribution License, which permits unrestricted use, distribution, and reproduction in any medium, provided the original work is properly cited.

\begin{abstract}
The interactive behaviors between transverse magnetic fields and axial loads of a magnetoelastic (ME) beam subjected to general boundary conditions are investigated. In particular, the instability criterion for the magneto-mechanical buckling problem is intricately discussed based on the structure characteristics and the initial conditions. The equation of motion for the proposed physical model is introduced according to the Hamilton's principle, and the stability criterion is obtained by using the method of multiple scales implemented on both spatial and time domains. Eventually a so-called Schrodinger equation with cubic nonlinearity (NLS) can be generated by suitably changing the variables; as a result, the stable criterion for the magnetoelastic beam can be acquired after dissecting the nonlinear Schrodinger equation and requiring the imaginary part of the time domain solution to be vanished. Stability criterion curve for the dispersion equation of the ME beam is firstly depicted in order to reveal the magnificent influence of the structure characteristic itself, followed by the instability constraint due to the variation of initial conditions and the observation locations. The results indicate that the prior one actually denotes a parabola, whereas the latter one is sometimes a diamond-like or ellipse-like region spotting along the prior one.
\end{abstract}

\section{Introductions}

The electromagnetic phenomena which arose from electrical machinery, communicating equipments, and computer chips have addressed wide attention in the past years due its significant role on human's daily life. and diamagnetic structures such as beams, plates, and shells are extensively employed in the modern electromagnetic equipments, and accordingly 
provide a real understanding for the mechanism of electricity and magnetism coexisting in the conventional elasticity.

Since the structures are often set in high magnetic fields, they are correspondingly subjected to strong magnetic forces resulting from the applied magnetic fields, however, the magnetic force not only causes the structural deformation but also respectively changes with the deformation itself. Therefore, when the induced magnetization is under consideration, the analysis of the magnetoelastic structures with multiphases coupling effects becomes more complicated, and indeed requires further examination either on the dynamical behavior or stability issues.

In this paper, the interactive behaviors between transverse magnetic fields and axial loads of a magnetoelastic beam subjected to general boundary conditions are investigated. In specific, the instability criterion for the magnetomechanical buckling problem is intricately discussed according to the structure characteristics as well as the initial conditions. The fundamental concepts and relations about the electro-magnetic theory are adopted in this paper based on the content written in [1], and some other systematic references for the theory of magnetoelastic solid mechanics found in [2] are also contained. Inspections on the behavior of interaction between magnetic field and mechanical deformation for the structures made of magnetoelastic materials have been conducted by many researchers and engineers. Some of them are briefly described in the next paragraph.

Moon and Pao [3] proposed a mathematical model for the buckling problem of a cantilever beam-plate in a transverse magnetic field with distributed magnetic forces and torques. Wallerstein and Peach [4] studied the magnetoelastic buckling of beams and plates with magnetically soft material. Miya et al. [5] investigated the magnetoelastic buckling of a cantilevered beam-plate by applying the experimental and finite element methods. Moon and Pao [6] presented the vibration and parametric instability of a cantilevered beam-plate in a transverse magnetic field and also provided the theoretical and experimental results. In Moon-Pao's theoretical analysis, the magnetic torque without axial load was considered; therefore, the axial load studied in this paper does not apply to their discussion. Kojima et al. [7] investigated the parameter nonlinear forced vibrations of a beam with a tip mass subjected to alternating electromagnetic forces acting on the tip mass. Shin et al. [8] have studied the transient vibrations of a simply supported beam with axial loads and transverse magnetic fields. Liu and Chang [9] performed the vibration analysis of a beam with general boundary conditions in a magnetic field subjected to axial load and external force by introducing the orthogonal characteristic polynomial. Wu [10] performed the analysis of dynamic instability and vibration motions of a pinned beam with transverse magnetic field and thermal loads. Pratiher and Dwivedy [11] studied the parametric instability of a cantilever beam with magnetic field and periodic axial load.

In view of the fact that the interactions among axial load, magnetic force, and magnetic couples are complicated, yet, important to the dynamical analysis of structural instability, a magnetoelastic (ME) beam system involving axial load, transverse magnetic field, and spring foundation is considered in the present paper. For simplicity, the axial force and transverse magnetic field are assumed to be static, that is, independent of time variable; however, this paper is aiming at finding out the stability criterions under which the magnetoelastic beam can be dynamically stabilized; therefore, it is suggested that neither the magnitude of axial force nor that of the transverse magnetic field should exceed the parameters restrained by the criterion even though they are set to be periodical or other else.

The equation of motion for the proposed physical model is introduced based on the authors' previous work, and some of the quantities related to magnetic field are evaluated 
by adopting reasonable assumptions and approximations. The stability criterion for an ME beam subjected to both transverse magnetic field and axial load can be obtained by using the method of multiple scales implemented on both spatial and time variables. After collecting the small perturbed parameter $\varepsilon$ with the same order, a set of relevant partial differential equations resulting from the perturbation process can thus be derived. Based on some assumptions dedicating to simplify the mathematical modeling of the whole system, the analytic solutions of the respective perturbed equations can be solved one by one successively. As a result of suitably changing the variables, a so-called Schrodinger equation with cubic nonlinearity (NLS) will be generated from the evaluation of perturbation equation for $O\left(\varepsilon^{3}\right)$. By sophisticated technique dissecting the above nonlinear Schrodinger equation, the criterion for stable condition to the whole system can be acquired by requiring the imaginary part of the time domain solution to be vanished.

\section{Formulations}

\subsection{Statement of the Problem}

In this paper, the physical model of a magnetoelastic (ME) beam system rested upon a Winkler-type foundation and subjected to axial applied force and transverse magnetic field as shown in Figure 1 is investigated. The beam is made of linearly magnetoelastic material with width $d$, thickness $h$, length $L$ and is subjected to an applied axial force $\vec{P}=P_{0} \vec{i}$ in the $x$ direction and a transverse magnetic field $\vec{B}=B_{0} \vec{j}$, linear viscous damper in the $y$-direction, and attached by the linear springs with constant $K$. In order to simplify the analysis, the proposed beam is assumed as Euler-Bernoulli type.

\subsection{Mathematical Modeling}

Hamilton's principle [8] is adopted to derive the equation of motion of the beam as follows:

$$
m \frac{\partial^{2} w}{\partial t^{2}}+C_{d} \frac{\partial w}{\partial t}+Y I \frac{\partial^{4} w}{\partial x^{4}}+K w+P \frac{\partial^{2} w}{\partial x^{2}}=\frac{\partial c}{\partial x}+\frac{\partial}{\partial x}\left[\left(\int_{0}^{x} p d \xi\right) \frac{\partial w}{\partial x}\right]
$$

where $w(x, t)$ denotes the transverse displacement of the middle plane, $m$ is the mass per unit length, $C_{d}$ is the damping coefficient, $Y$ is the Young's modulus, $I$ is the moment of inertia of the cross section, $K$ is the constant of spring, $P$ is the axial load per unit length along $x$ direction, $c$ represents the induced couple per unit length due to the existence of magnetic field, and $p$ is the body force per unit length contributed by the magnetic force.

For the dia- and para-magnetoelastic material, the magnetization $\mathbf{M}$ of the medium due to the existence of auxiliary magnetic field $\mathbf{H}$ can be defined as $\mathbf{M}=\chi_{m} \mathbf{H}$ and $\mathbf{H}$ can be determined by the relation $\mathbf{B}=\mu_{0} \mathbf{H}$ with $\mathbf{B}$ being the externally applied magnetic field. Thus the induced couple or magnetic torque can be read as

$$
\mathbf{c}=\int(\mathbf{M} \times \mathbf{B}) \mathbf{d V},
$$




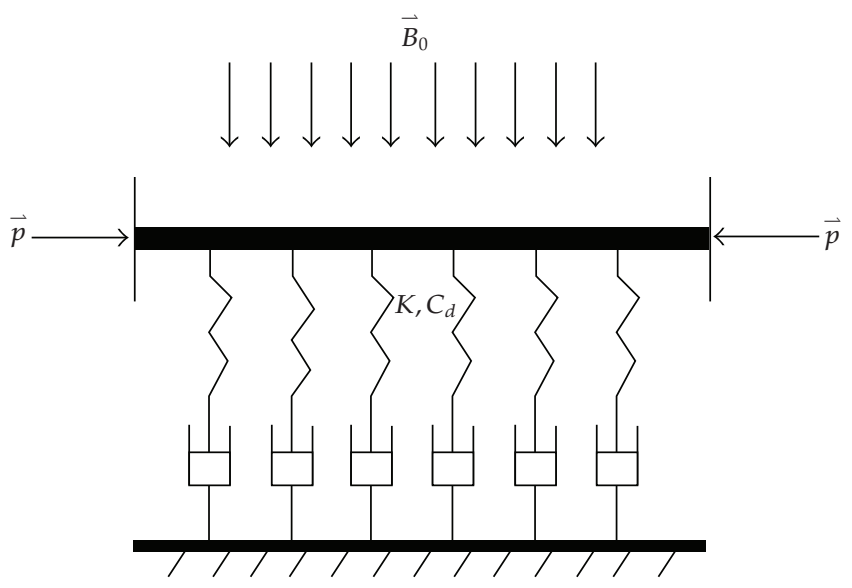

Figure 1: Physical model for a magnetoelastic (ME) beam subjected to axial load and magnetic field.

in which $\chi_{m} \equiv\left(\mu_{0}-\mu\right) / \mu_{0}=1-\mu_{r}$ is the magnetic susceptibility with $\mu$ being the material permeability and $\mu_{0}$ the permeability of free space. The magnetization $\mathbf{M}$ can be rewritten as $\mathbf{M}=\left(\chi_{m} / \mu_{0}\right) \mathbf{B}=\left(\chi_{m} / \mu_{0}\right)\left|B_{0}\right| \vec{n}$ and is also called volume density of magnetic moment, meanwhile, the magnetic field $\mathbf{B}$ is sometimes called the magnetic displacement or the induced magnetic field.

According to the small deformation theory, it can be shown [9] that the velocity term, $\dot{x}$, for the magnetoelastic beam can be simplified into

$$
\dot{x} \equiv \frac{d x}{d t} \approx-\int_{0}^{x} \frac{\partial w}{\partial \xi} \frac{\partial^{2} w}{\partial t \partial \xi} d \xi
$$

As a result, the component of the body force contributed by the magnetic field $\mathbf{B}$ can be written as

$$
p=\text { component } \int \sigma\left(\overrightarrow{\dot{r}} \times \vec{B}_{0}\right) \times \vec{B}_{0} d V=\sigma B_{0}^{2} h d \frac{d x}{d t}=-\sigma B_{0}^{2} h d \int_{0}^{x} \frac{\partial w}{\partial \xi} \frac{\partial^{2} w}{\partial t \partial \xi} d \xi
$$

Mean while, the component of the magnetic couple induced by the applied magnetic field can be expressed as

$$
c=\text { component } \int \vec{M} \times \vec{B}_{0} d V=M B_{0} h d \sin \theta \approx M B_{0} h d \theta=M B_{0} h d \frac{\partial w}{\partial x},
$$

in which $\sigma$ is the electrical conductivity of the ME beam and $h$ and $d$ represent the thickness and the depth of the beam, respectively.

\subsection{Perturbation and Multiscale Method}

For the nonlinear differential system stated in (2.1), the solutions in a form of harmonic wave propagation are permitted, however, with the restriction that the bending wave is slowly 
changed in spatial and time domain than the carrying wave [12]. Therefore, it is fairly feasible to practice the solution of (2.1) in the following form at the vicinity of the equilibrium state:

$$
w=\varepsilon w_{1}+\varepsilon^{2} w_{2}+\varepsilon^{3} w_{3}+\cdots,
$$

in which the small perturbed parameter can be taken as $\varepsilon \equiv \lambda_{c} / \lambda_{e} \ll 1$ with $\lambda_{c}$ and $\lambda_{e}$ indicating the wave lengths of carrying and bending waves, respectively.

By adopting $x$ and $t$ as usual space and time variables for carrying wave, and performing the method of multiple scales, a set of "slow" time and space variables: $T_{n}=\varepsilon^{n} t$, $X_{n}=\varepsilon^{n} x$, are introduced in the present study. Due to the principle of chain rule, the operators of differentiation $\partial / \partial x$ and $\partial / \partial t$ in (2.1) should be accordingly modified in order to account for the dependence of "slow" variables $T_{n}$ and $X_{n}$ on regular $x$ and $t$ variables; therefore, these derivatives are transformed into the following operators:

$$
\begin{gathered}
\frac{\partial}{\partial x} \equiv \frac{\partial}{\partial X_{0}}+\sum_{n=1} \varepsilon^{n} \frac{\partial}{\partial X_{n}} \\
\frac{\partial}{\partial t} \equiv \frac{\partial}{\partial T_{0}}+\sum_{n=1} \varepsilon^{n} \frac{\partial}{\partial T_{n}}
\end{gathered}
$$

It should be noted that the variables $X_{0} \equiv \varepsilon^{0} x=x, X_{n}, T_{0} \equiv \varepsilon^{0} t=t$, and $T_{n}$ stated above are all independent variables with respect to each other, and, for the sake of briefness, the variables $T_{0}$ and $X_{0}$ will be replaced by $x$ and $t$ correspondingly in the following derivations and for the rest of the paper.

Substituting (2.6) and (2.7) into (2.1) and equating the terms with the same order of $\varepsilon$, one can obtain

$$
\begin{aligned}
O(\varepsilon): \quad L_{0} w_{1}= & 0, \quad L_{0} \equiv m \frac{\partial^{2}}{\partial t^{2}}+C_{d} \frac{\partial}{\partial t}+Y I \frac{\partial^{4}}{\partial x^{4}}+K+\left(P-M B_{0} h d\right) \frac{\partial^{2}}{\partial x^{2}} \\
O\left(\varepsilon^{2}\right): \quad L_{0} w_{2}= & -2 m \frac{\partial^{2} w_{1}}{\partial t \partial T_{1}}-C_{d} \frac{\partial w_{1}}{\partial T_{1}}-4 Y I \frac{\partial^{2} w_{1}}{\partial^{3} x \partial X_{1}}-2\left(P-M B_{0} h d\right) \frac{\partial^{2} w_{1}}{\partial x \partial X_{1}} \\
O\left(\varepsilon^{3}\right): \quad L_{0} w_{3}= & -m\left(2 \frac{\partial^{2} w_{1}}{\partial t \partial T_{2}}+\frac{\partial^{2} w_{1}}{\partial T_{1}^{2}}+2 \frac{\partial^{2} w_{2}}{\partial t_{0} \partial T_{1}}\right)-C_{d}\left(\frac{\partial w_{2}}{\partial T_{1}}+\frac{\partial w_{1}}{\partial T_{2}}\right) \\
& -Y I\left[4 \frac{\partial^{4} w_{1}}{\partial x_{0}^{3} \partial X_{2}}+6 \frac{\partial^{4} w_{1}}{\partial x^{2} \partial X_{1}^{2}}+4 \frac{\partial^{4} w_{2}}{\partial x^{3} \partial X_{1}}\right] \\
& -\left(P-M B_{0} h d\right)\left(2 \frac{\partial^{2} w_{1}}{\partial x_{0} \partial X_{2}}+\frac{\partial^{2} w_{1}}{\partial X_{1}^{2}}+2 \frac{\partial^{2} w_{2}}{\partial x \partial X_{1}}\right) \\
& -\left(\sigma B_{0}^{2} h d\right)\left(\int_{0}^{x_{0}} \frac{\partial w_{1}}{\partial \xi} \frac{\partial^{2} w_{1}}{\partial t \partial \xi} d \xi \cdot \frac{\partial w_{1}}{\partial x_{0}}\right) \\
& -\left(\sigma B_{0}^{2} h d\right)\left(\int_{0}^{x_{0}} \int_{0}^{\xi} \frac{\partial w_{1}}{\partial \eta} \frac{\partial^{2} w_{1}}{\partial t \partial \eta} d \eta d \xi \cdot \frac{\partial^{2} w_{1}}{\partial x_{0}^{2}}\right)
\end{aligned}
$$


It is noticed that (2.8) is actually a homogenous version of the governing equation, say (2.1), and one can easily express the solution of (2.8) in the form of harmonic waves with the amplitude depending on slow variables, that is,

$$
\begin{gathered}
w_{1}=A\left(X_{1}, T_{1}, X_{2}, T_{2}, \ldots\right) \exp (i \theta)+\text { c.c. } \\
\theta=k x-\omega t
\end{gathered}
$$

wherein c.c. stands for the complex conjugate and $\theta$ can be thought as the phase angle of the propagating wave. To assure that the net axial load appeared in (2.8), say $\left(P-M B_{0} h d\right)$, is of the compressive type, it is required that this term should be positive in the present study.

According to (2.8), the dispersion equation will have the following form:

$$
m \omega^{2}+i C_{d} \omega+\left[Y I k^{4}-\left(P-M B_{0} h d\right) k^{2}+K\right]=0
$$

and after imposing implicit differentiation with respect to $k$ on the dispersion equation and suppose $\omega=\omega(k)$, the relation between $\omega$ and $k$ can be expressed implicitly as

$$
\frac{d \omega}{d k}=\frac{4 Y I k^{3}-2 k\left(P-M B_{0} h d\right)}{2 m \omega+i C_{d}} .
$$

Substituting (2.11) into (2.9) gives

$$
L_{0} w_{2}=e^{i \theta}\left\{\frac{\partial A}{\partial T_{1}}\left[2 m \omega i-C_{d}\right]+i \frac{\partial A}{\partial X_{1}}\left[4 Y I k^{3}-2\left(P-M B_{0} h d\right) k\right]\right\}+\text { c.c. }
$$

The first term in (2.14) is secular term or sometimes called the small divisor term, and for the applicability of theory for excitation, we should demand the elimination of this term, that is,

$$
\frac{\partial A}{\partial T_{1}}[2 m \omega i-C d]+i \frac{\partial A}{\partial X_{1}}\left[4 Y I k^{3}-2\left(P-M B_{0} h d\right) k\right] \equiv 0
$$

Since no other term left in (2.14) after imposing (2.15), there is no particular solution as a result, the general solution of (2.14) thus can be determined to be identical with the homogeneous solution, that is,

$$
w_{2}=A\left(X_{1}, T_{1}, X_{2}, T_{2}\right) e^{i \theta}=w_{1}
$$


By taking the same token, substituting (2.11) and (2.16) into (2.10) and demanding the vanish of secular terms will result in the following expression:

$$
\begin{aligned}
& (2 m i \omega-C d) \frac{\partial A}{\partial T_{2}}+(-m) \frac{\partial^{2} A}{\partial T_{1}^{2}}+\frac{\partial A}{\partial X_{2}}\left[-4 Y I(i k)^{3}-2\left(P-M B_{0} h d\right)(i k)\right] \\
& \quad+\frac{\partial^{2} A}{\partial X_{1}^{2}}\left[-6 Y I(i k)^{2}-\left(P-M B_{0} h d\right)\right] \\
& \quad-A|A|^{2}\left\{\left(\sigma B_{0}^{2} h d\right)\left[(i k)^{3}(-i \omega) \frac{e^{2 i \theta}-e^{-2 i \omega t}}{2 i k}+(i k)^{4}(-i \omega) \frac{1}{2 i k}\left(\frac{e^{2 i \theta}-e^{-2 i \omega t}}{2 i k}-\frac{x}{e^{2 i \omega t}}\right)\right]\right\} \\
& \quad=0 .
\end{aligned}
$$

Meanwhile, by taking differentiation on (2.15) with respect to $k$, we have

$$
\frac{\partial A}{\partial T_{1}}\left(m i \frac{d \omega}{d k}\right)+i \frac{\partial A}{\partial X_{1}}\left[6 Y I k^{2}-\left(P-M B_{0} h d\right)\right]=0
$$

furthermore, differentiating (2.18) with respect to $T_{1}$ gives

$$
\frac{\partial^{2} A}{\partial T_{1}^{2}}\left[-m \frac{d \omega}{d k}\right]-\frac{\partial^{2} A}{\partial T_{1} X_{1}}\left[6 Y I k^{2}-\left(P-M B_{0} h d\right)\right]=0
$$

similarly, differentiating (2.18) with respect to $X_{1}$ gives

$$
\frac{\partial^{2} A}{\partial X_{1} \partial T_{1}}\left[m i \frac{d \omega}{d k}\right]+i \frac{\partial^{2} A}{\partial X_{1}^{2}}\left[6 Y I k^{2}-\left(P-M B_{0} h d\right)\right]=0
$$

Adding the above two equations gets

$$
\frac{\partial^{2} A}{\partial T_{1}^{2}}(-m)+\frac{\partial^{2} A}{\partial X_{1}^{2}}\left[6 Y I k^{2}-\left(P-M B_{0} h d\right)\right]=\frac{\partial^{2} A}{\partial T_{1} \partial X_{1}}\left[\frac{6 Y I k^{2}-\left(P-M B_{0} h d\right)}{d \omega / d k}-m \frac{d \omega}{d k}\right]
$$

also we have the following results after taking derivative with respect to $X_{1}$ on (2.15):

$$
\frac{\partial^{2} A}{\partial T_{1} \partial X_{1}}=-\frac{\partial^{2} A}{\partial X_{1}^{2}} \frac{4 Y I k^{3}-2\left(P-M B_{0} h d\right) k}{2 m \omega+i C d}=-\frac{\partial^{2} A}{\partial X_{1}^{2}} \frac{d \omega}{d k}
$$


thus (2.21) becomes

$$
\frac{\partial^{2} A}{\partial T_{1}^{2}}(-m)+\frac{\partial^{2} A}{\partial X_{1}^{2}}\left[6 Y I k^{2}-\left(P-M B_{0} h d\right)\right]=-\frac{\partial^{2} A}{\partial X_{1}^{2}}\left[6 Y I k^{2}-\left(P-M B_{0} h d\right)-m\left(\frac{d \omega}{d k}\right)^{2}\right] .
$$

Therefore, (2.17) can be reduced into

$$
\begin{aligned}
& (2 m i \omega-C d) \frac{\partial A}{\partial T_{2}}+\frac{\partial A}{\partial X_{2}}\left[-4 Y I(i k)^{3}-2\left(P-M B_{0} h d(i k)\right)\right] \\
& -\frac{\partial^{2} A}{\partial X_{1}^{2}}\left[6 Y I k^{2}-\left(P-M B_{0} h d\right)-m\left(\frac{d \omega}{d k}\right)^{2}\right]+A|A|^{2}[f(x, t)]=0,
\end{aligned}
$$

where

$$
f(x, t) \equiv-\left(\sigma B_{0}^{2} h d\right)\left[(i k)^{3}(-i \omega) \frac{e^{2 i \theta}-e^{-2 i \omega t}}{2 i k}+(i k)^{4}(-i \omega) \frac{1}{2 i k}\left(\frac{e^{2 i \theta}-e^{-2 i \omega t}}{2 i k}-\frac{x}{e^{2 i \omega t}}\right)\right] .
$$

In order to eliminate some variables, we now introduce a new variable $\bar{T}$ as

$$
\bar{T} \equiv T_{2}+\frac{d k}{d \omega} X_{2}
$$

which implies

$$
\frac{\partial}{\partial \bar{T}} \equiv \frac{\partial}{\partial T_{2}} \frac{\partial T_{2}}{\partial \bar{T}}+\frac{\partial}{\partial X_{2}} \frac{\partial X_{2}}{\partial \bar{T}}=\frac{\partial}{\partial T_{2}}+\frac{d \omega}{d k} \frac{\partial}{\partial X_{2}}
$$

With the differential operator defined in (2.27), we can rewrite the first two terms in (2.24), say

$$
(2 m i \omega-C d) \frac{\partial A}{\partial T_{2}}+\frac{\partial A}{\partial X_{2}}\left[4 Y I i k^{3}-2 i k\left(P-M B_{0} h d\right)\right]
$$

into the following expression in term of $\bar{X}$ :

$$
(2 m i \omega-C d)\left[\frac{\partial A}{\partial T_{2}}+\frac{d \omega}{d k} \frac{\partial A}{\partial X_{2}}\right]=(2 m i \omega-C d) \frac{\partial A}{\partial \bar{T}} .
$$


Therefore, the following Schrodinger-type equation can then by obtained as a result

$$
i \frac{\partial A}{\partial \bar{T}}=\frac{6 Y I k^{2}-\left(P-M B_{0} h d\right)-m(d \omega / d k)^{2}}{2 m \omega+i C d} \frac{\partial^{2} A}{\partial X_{1}^{2}}+\frac{[f(x, t)]}{2 m \omega+i C d} A|A|^{2},
$$

which can be simplified as

$$
i \frac{\partial A}{\partial \bar{T}}=\beta \frac{\partial^{2} A}{\partial X_{1}^{2}}+\gamma A|A|^{2}
$$

where $\beta$ and $\gamma$ are complex, and can be expressed as follows:

$$
\begin{gathered}
\beta \equiv \frac{6 Y I k^{2}-\left(P-M B_{0} h d\right)-m(d \omega / d k)^{2}}{2 m \omega+i C d} \\
\gamma \equiv \frac{-\left(\sigma B_{0}^{2} h d\right)}{2 m \omega+i C d}\left[(i k)^{3}(-i \omega) \frac{e^{2 i \theta}-e^{-2 i \omega t}}{2 i k}+(i k)^{4}(-i \omega) \frac{1}{2 i k}\left(\frac{e^{2 i \theta}-e^{-2 i \omega t}}{2 i k}-\frac{x}{e^{2 i \omega t}}\right)\right] .
\end{gathered}
$$

By now we have the Schrodinger equation with complex coefficient as mentioned above; if the viscosity is neglected $\left(C_{d}=0\right)$, then the Schrodinger-type equation with real coefficients can then be obtained.

\subsection{Stable Criterion for Schrodinger Equation}

Equation (2.31) describes a behavior of modulated waves and is commonly referred to as the nonlinear Schrodinger (NLS) equation with cubic nonlinearity. Even though it has been proved that there is a solution for the NLS equation with real coefficient [13], however, the complex coefficients for NLS equation as stated in (2.31) make the analysis become more difficult, yet no related report can be found up to date.

In the following, an attempt to find the stable criterion for the NLS equation as stated in (2.31) is conducted; as a first step, we can express the solution for $A\left(X_{1}, \bar{T}\right)$ in the following form:

$$
A\left(X_{1}, \bar{T}\right)=A_{0} \exp (i \Theta)=A_{0} \exp \left[i\left(\kappa X_{1}-\Omega \bar{T}\right)\right]
$$

Substituting it into (2.31) leads to

$$
\Omega=-\beta \kappa^{2}+\gamma A_{0}^{2}
$$


thus we can have

$$
A\left(X_{1}, \bar{T}\right)=A_{0} \exp \left\{i\left[\kappa X_{1}+\left(\beta \kappa^{2}-\gamma A_{0}^{2}\right) \bar{T}\right]\right\}=A_{0} \exp \left(i \kappa X_{1}\right) \cdot \exp \left[i\left(\beta \kappa^{2}-\gamma A_{0}^{2}\right) \bar{T}\right] .
$$

For the above solution to be stable in the time domain, it is required that the imaginary part of the frequency should be greater than zero; therefore, the stability criterion would be given by the following inequality:

$$
\operatorname{Im}\left(\beta \kappa^{2}-\gamma A_{0}^{2}\right)>0
$$

where $A_{0}$ can be indicated by the amplitude given in the initial conditions.

However, as we can detect from (2.32) and (2.33) that the value for $\beta$ is purely real if damping coefficient $C_{d}$ is neglected, and the value for $\gamma$ is definitely complex with strong dependence on the slow variables, namely, $x$ and $t$. This phenomenon suggests that the criterion for stability should be determined not only by the beam characteristics but also by the initial conditions it was provoked by, namely, the catching time $t$ and the observation location $x$ as well as the initial amplitude $A_{0}$. In the present study, the initial conditions $(t=0)$ at a specific location $\left(x=x_{0}\right)$ will be accordingly imposed in order to see the spectacular influence caused by the value of $\gamma$, and by substituting related values into the inequality in (2.37), the diagrams for the stability region subjected to various beam characteristics can be depicted.

It should be noted that (2.37) shows how initial conditions can affect the stability of the wave propagation; nevertheless, the stable criterion for the whole system, say the differential equation, is actually dominated by the dispersion equation stated in (2.12). Therefore, as far as the stability problem is concerned, we should first examine the stability region for the dispersion equation with respect to different boundary conditions, and then carefully point out the instability criterions caused by the initial conditions as well as the observation location, which are expected to be some subdomains among the prior one or just on the border.

\section{Numerical Examples}

For the purpose of demonstration, a low-carbon steel is considered in this study, of which the material constants [9] are respectively $Y=194 \mathrm{GPa}, m=0.03965 \mathrm{~kg} / \mathrm{m}, L=0.5 \mathrm{~m}, h=$ $0.005 \mathrm{~m}, d=0.001 \mathrm{~m}, \mu_{r}=1.00001, \mu_{0}=4 \pi \times 10^{-7} \mathrm{Hm}^{-1}, \sigma=10^{7} \mathrm{Sm}^{-1}$, and $K=1.0 \mathrm{~N} / \mathrm{m}$. To account for the effects of both initial conditions and boundary conditions on the stability criterion of the ME beam, several cases involving various combinations of these conditions have been examined. For the convenience of comparison, the catching time for all cases is set to be $t=0$ whereas the magnitude of initial displacement is set to the same with the beam thickness, that is, $A_{0}=h$ is chosen for all stability discussions in following examples.

Four kinds of commonly seen boundary conditions are implemented; they are, respectively, simply supported on both end (S-S), fixed at left and simply supported at right (C-S), cantilever beam (C-F), and fixed on both ends (C-C). The wave numbers with respect to the corresponding boundary conditions are stated is Table 1 as a reference despite the fact 
Table 1: The first three wave numbers corresponding to various boundary conditions.

\begin{tabular}{lccc}
\hline Boundary condition & & Mode number & $N=3$ \\
\hline S-S & $\frac{\pi}{L}$ & $N=2$ & $\frac{2 \pi}{L}$ \\
C-S & $\frac{3.926602}{L}$ & $\frac{7.068583}{L}$ & $\frac{10.210176}{L}$ \\
C-F (Cantilever) & $\frac{1.875104}{L}$ & $\frac{4.694091}{L}$ & $\frac{7.854757}{L}$ \\
C-C & $\frac{4.730041}{L}$ & $\frac{7.853205}{L}$ & $\frac{10.995607}{L}$ \\
\hline
\end{tabular}

${ }^{*} \mathrm{~S}$ stands for Simply supported, $\mathrm{C}$ is for clamped, $\mathrm{F}$ is for free end

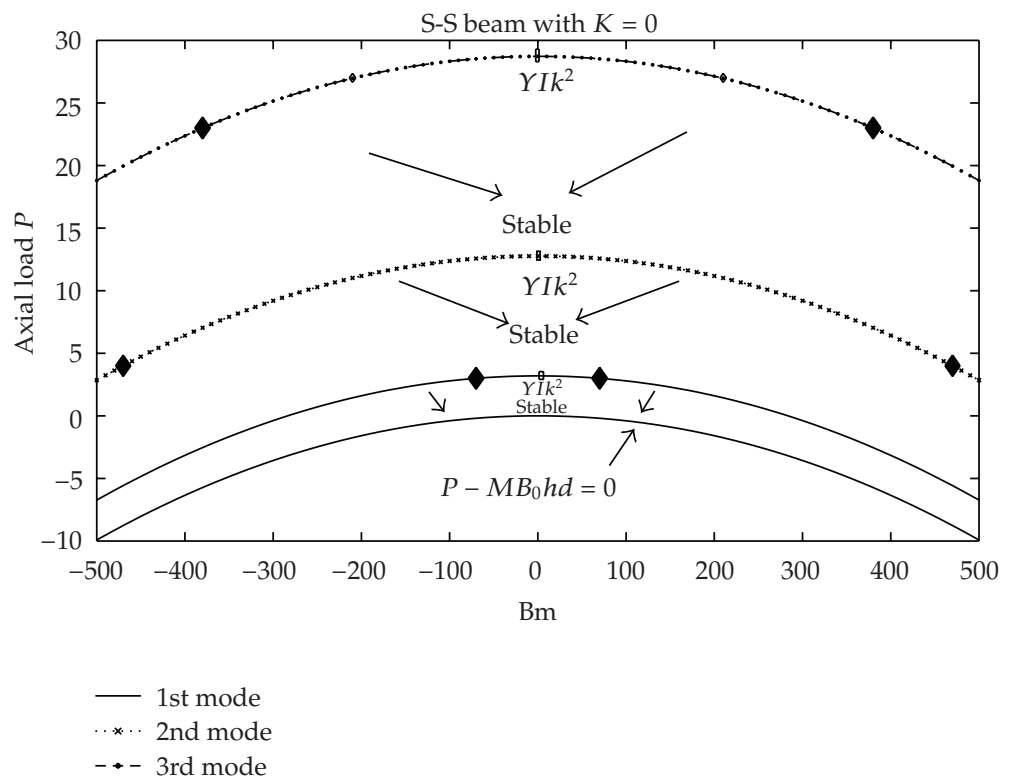

Figure 2: Stability Region for an ME beam with simply supported boundary conditions subjected to axial load and magnetic; with no spring support.

that they can still be found in any textbook related to the structure vibration problems. Due to the different beam characteristics with respect to different boundary conditions, the location for stable observation is properly pinpointed according to the imposed boundary conditions for each specific case. The observation location $x_{0}$ for the stability criterion of a cantilever beam is pinpointed at the right end $\left(x_{0}=L\right)$ of the ME beam; however, for the other cases, the location is fixed to be at the center point of the beam length, that is, $x_{0}=L / 2$.

Figure 2 presents the stability region for a magnetoelastic beam with S-S boundary conditions subjected to axial external force and transverse magnetic field; the effect of spring foundation is here neglected. Three modes of wave number are calculated; magnetic field is ranging from -500 Tesla to 500 Tesla whereas the axial external force is from $-10 \mathrm{~N}$ to $30 \mathrm{~N}$. The signs for magnetic fields and axial forces simply indicate the direction these forces are applying to, that is, positive axial force means compression while negative implies tension, 


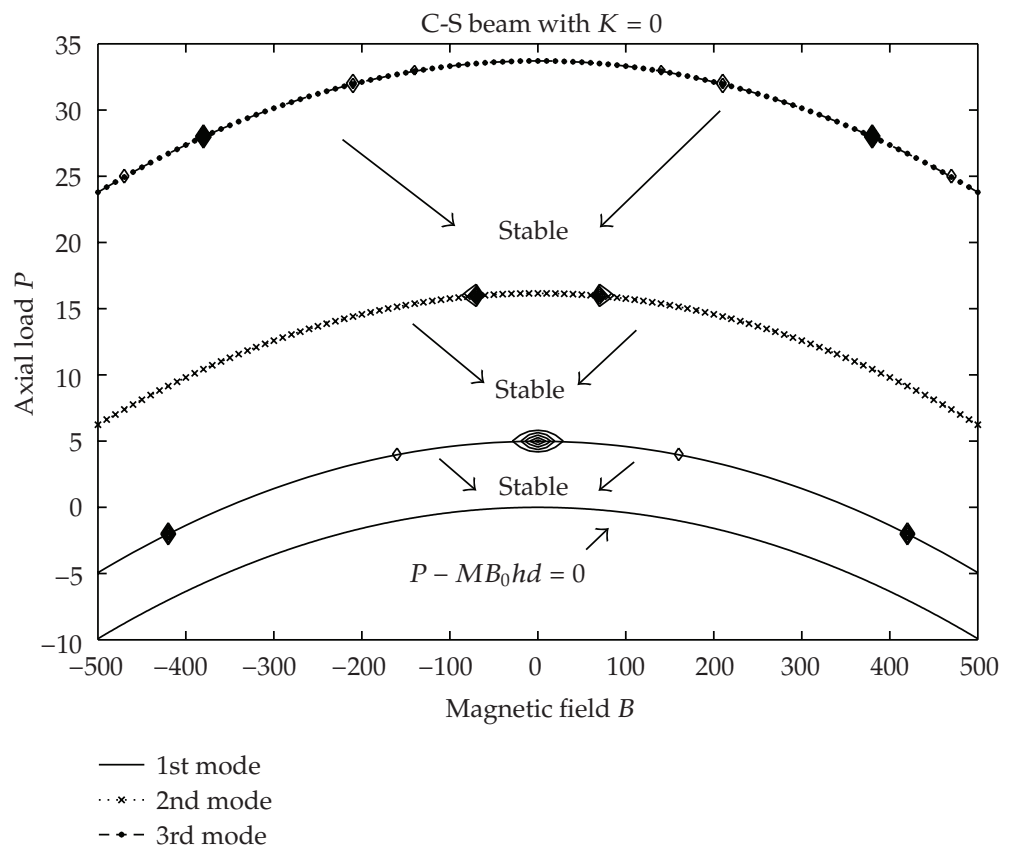

Figure 3: Stability Region for an ME beam with clamped-hinged boundary conditions subjected to axial load and magnetic field with no spring support.

positive magnetic field represents upward, and negative denotes downward. As it can be detected from Figure 2, the stability criterion curve for the dispersion equation, say (2.12), is indeed a parabola, and the instability constraint due to the initial condition, say (2.33), is presented in the shaded region, in which the parameters reside will incur imaginary parts of (2.37) to be negative. It should be noted that due to the numerical discreteness, the latter one looks like a shape of diamond; however, it is not necessarily a diamond and might be a circle or ellipse.

Figures 3-5 demonstrate the stability regions for a magnetoelastic beam with other boundary conditions subjected to the same setting as in Figure 2. Parabola for criterion curve can be observed again and the shaded regions are sometimes replaced by the lined regions, which indicate that the parameters for instability might not be compact on a bounded zone. By the fact that the strong instability due to the initial conditions and observation points makes the analysis become more complicated, a slight change in the number of shaded regions can be noticed, and a new added ellipse centered in the vicinity of zero magnetic field is detected. This incident reminds us that even though no external magnetic field is applied, that is, pure buckling problem for the ME beam is under consideration, yet we must be careful on examining the initial conditions in addition to the buckling criteria, so that accidentally invoking the instability of the system can be avoided. However, due to the distinct natures with respect to different boundary conditions, the aforementioned instability ellipses can be found to be centered along different modes of dispersion curves.

As it can be seen from Figure 3, the instability ellipse is center at the dispersion curve of the first mode for an ME beam with clamped-hinged boundary conditions. Also it is noticed that the instability diamonds become more frequently appeared along the curves than the simply supported case. Nevertheless, unlike the clamped-hinged case, the instability ellipses 


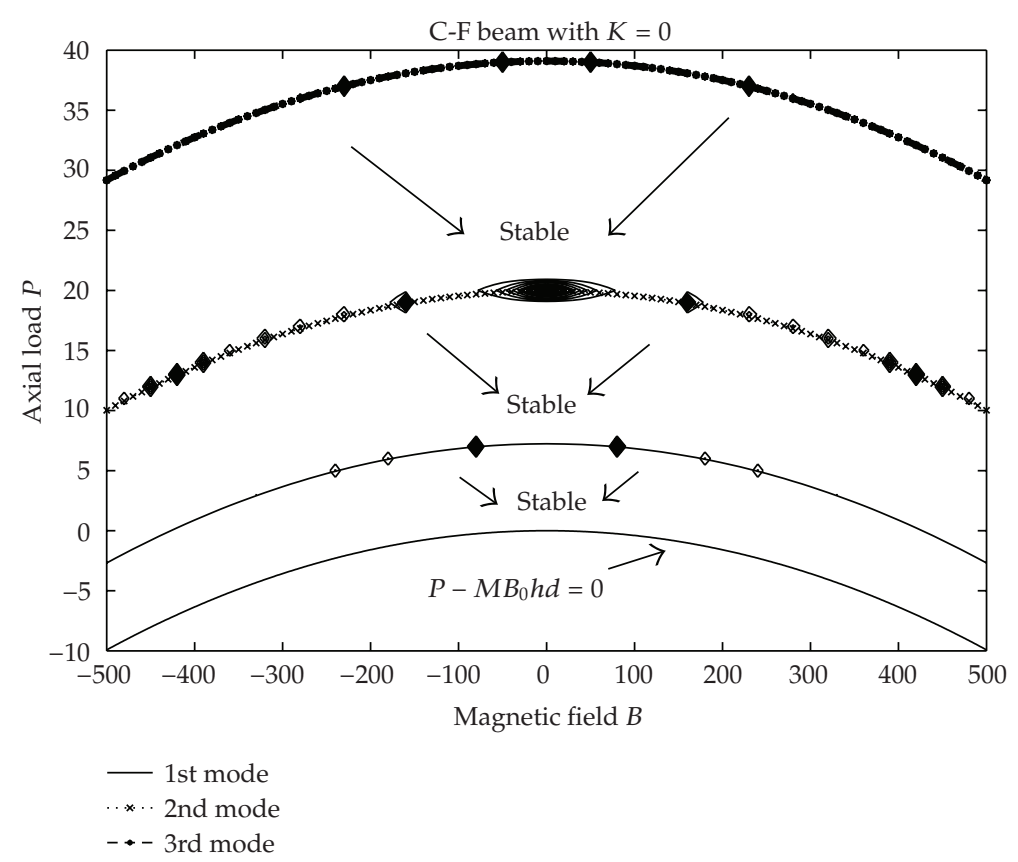

Figure 4: Stability Region for an ME beam with cantilever boundary conditions subjected to axial load and magnetic field with no spring support.

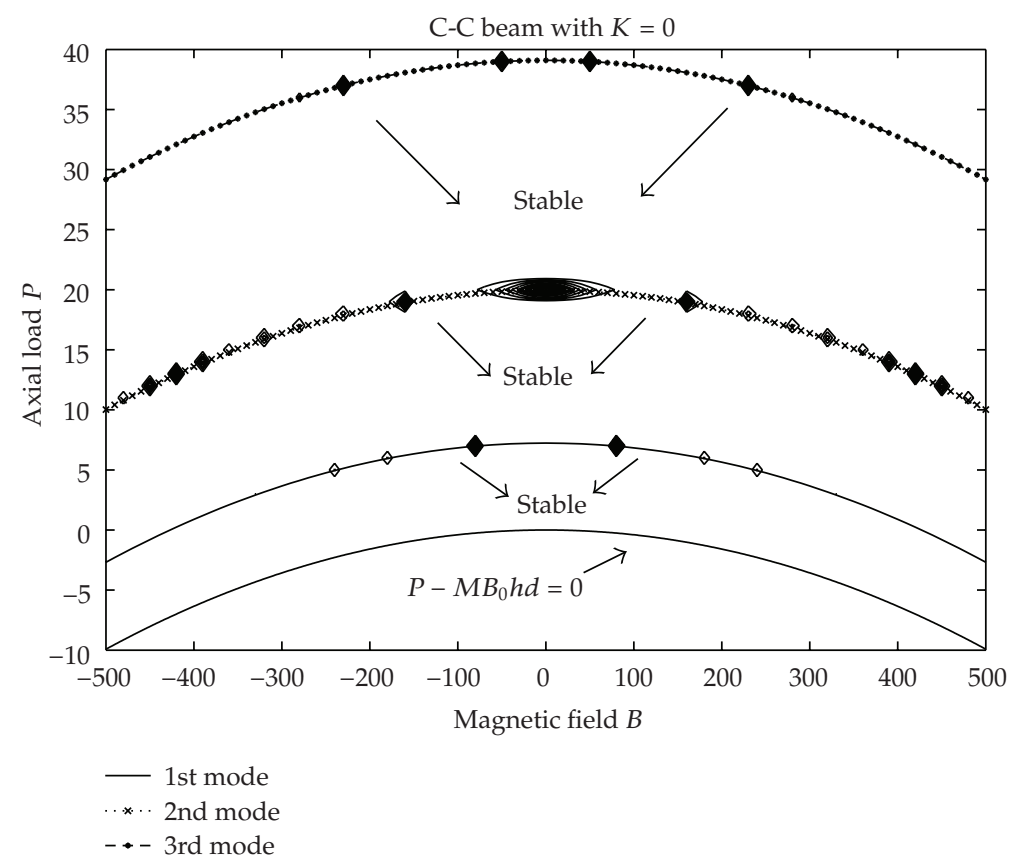

Figure 5: Stability Region for an ME beam with fixed-fixed boundary conditions subjected to axial load and magnetic field with no spring support. 


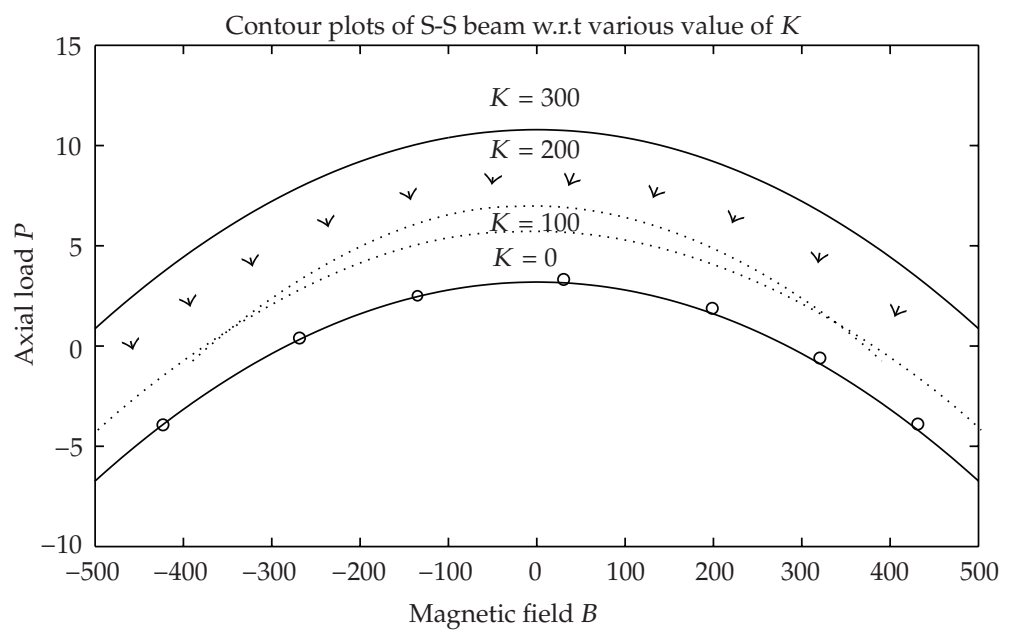

Figure 6: Stability Region for an ME beam with simply supported boundary conditions subjected to axial load and magnetic field with various spring constants.

for the cantilever ME beam, see Figure 4, can be found to seat on the dispersion curve of the third mode. In particular, the number of instability diamond is dramatically soaring so that almost every mode possesses at least 8 of the particular zones. Similar situation can also be observed in the case of fixed-fixed ME beam as shown in Figure 5, except that a remarkable instability ellipse resides on the dispersion curve of the second mode. It seems that on the curve of the second mode the instability restraints due to the initial conditions are compactly revealed, that is, we should put more attention when dealing with the instability problem for the second mode than the others.

Figure 6 demonstrates the dispersion curves of a simply supported ME beam subjected to external axial load and magnetic field with respect to various spring constant as foundation. As we expected, the larger value of spring constant results in the wider stability region to the ME beam system, that is, spring foundation plays a role in stabilizing the system, that is, if some certain axial load and magnetic field are necessarily imposed on the EM beam system, one way to promote the stability region, or to avoid instability, is to put the system on the Wrinkler-type foundation, thus system stability can still be retained.

At last, in order to verify the correctness of the results obtained by using the proposed method, the time responses of the system are presented for the stable and unstable regions individually. If we take simply supported ME beam as an example, adopting sinusoidal functions to be the mode shapes of the system, a set of temporal equations can be achieved after imposing Galerkin's procedure on (2.1); therefore, by performing Runge-Kutta method, the time responses of the system at middle point of the beam can be carried out for any desired modes.

Figure 7 depicts the ratio of displacement to beam thickness when the magnetic field and axial load are taken from the stable region as shown in Figure 2, while Figure 8 shows the results taken from the unstable region as a comparison. As it can be detected from Figure 7, when $B=10$ Tesla and $P=3.0 \mathrm{~N}$, the time responses of the system for the first, second, and third modes are plotted and the total deflection is calculated, all of which can be found to be of stable state. On the other hand, as $B=10$ Tesla and $P=4.0 \mathrm{~N}$ are chosen in Figure 8 , the 


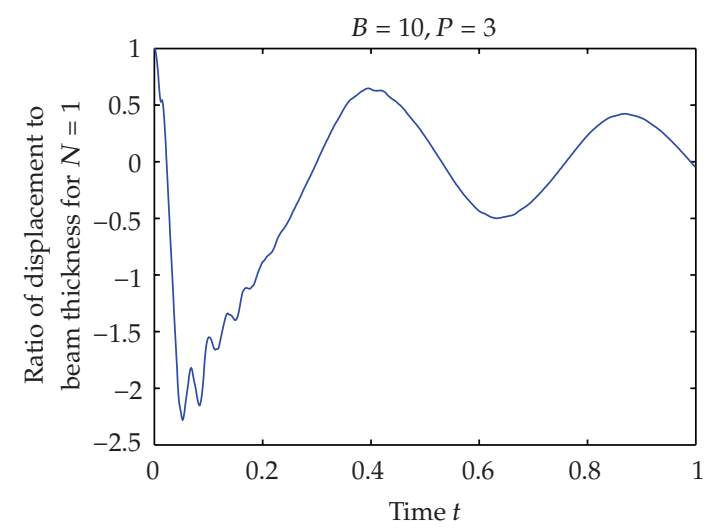

(a)

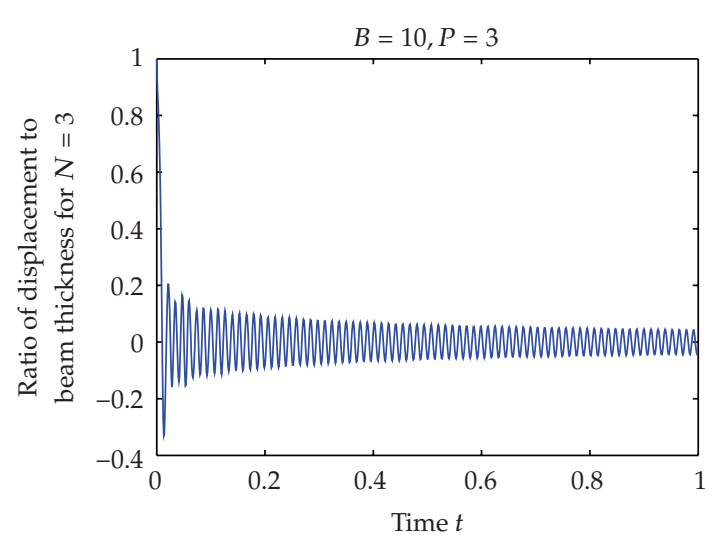

(c)

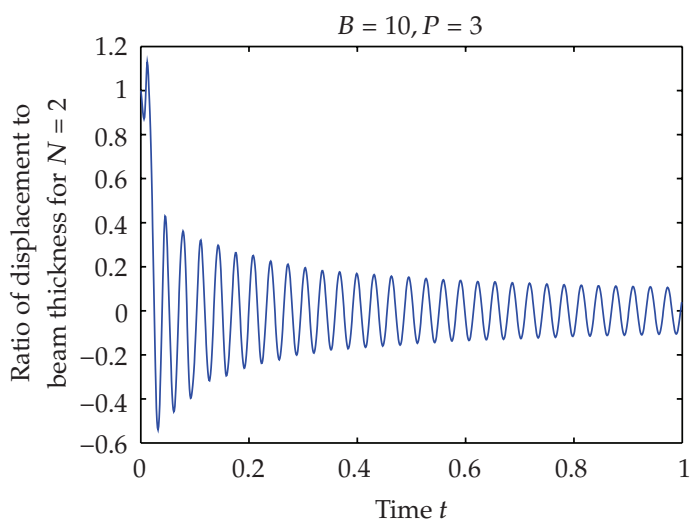

(b)

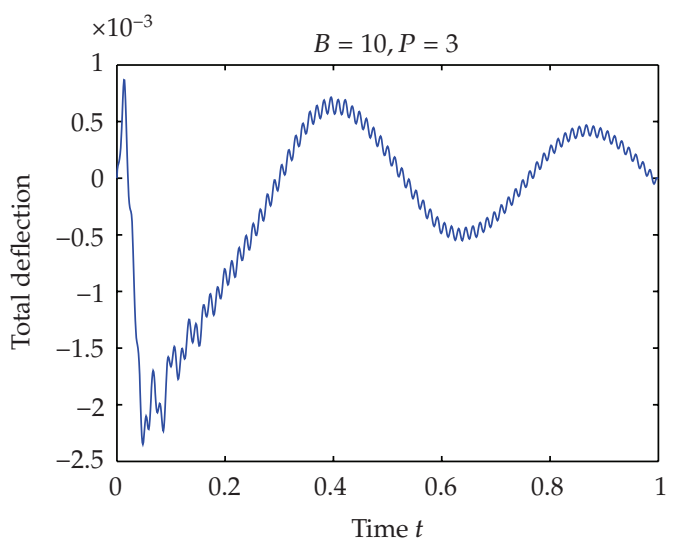

(d)

Figure 7: Transit response for an ME beam with simply supported boundary conditions subjected to axial load and magnetic field within stable region.

time response for the first mode is divergent, and those for the second and third modes are convergent; thus the total deflection is unstable as a result.

\section{Conclusions}

In this paper, the interactive behaviors between transverse magnetic fields and axial loads of a magnetoelastic $(\mathrm{ME})$ beam subjected to general boundary conditions are investigated. In particular, the instability criterion for the magneto-mechanical buckling problem is intricately discussed according to the structure characteristics as well as the initial conditions. The equation of motion for the proposed physical model is introduced based on the Hamilton's principle, and the stability criterion is obtained by using the method of multiple scales implemented on both spatial and time domains. By performing the perturbation procedure, a set of relevant partial differential equations can thus be derived with respect to different collecting order; meanwhile the analytic solutions for the respective perturbed equations can be resolved order by order successively based on some simplifications. Eventually a so-called Schrodinger equation with cubic nonlinearity (NLS) can be generated by suitably changing 


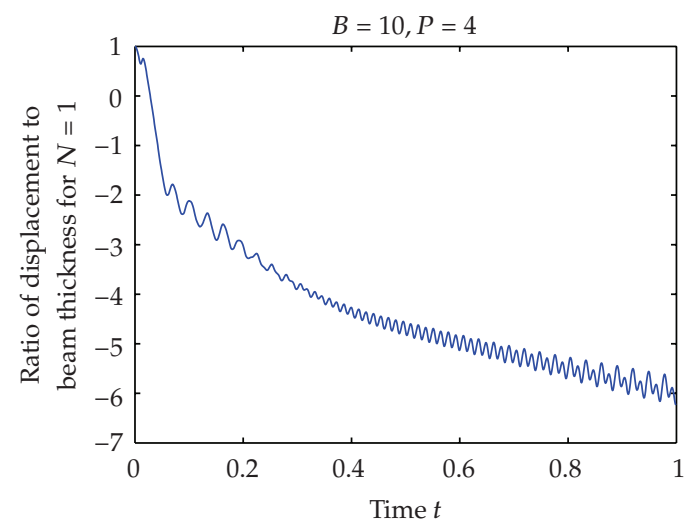

(a)

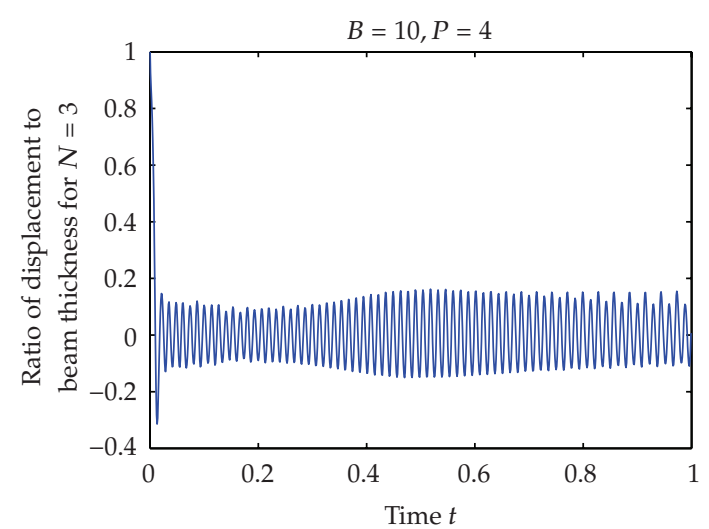

(c)

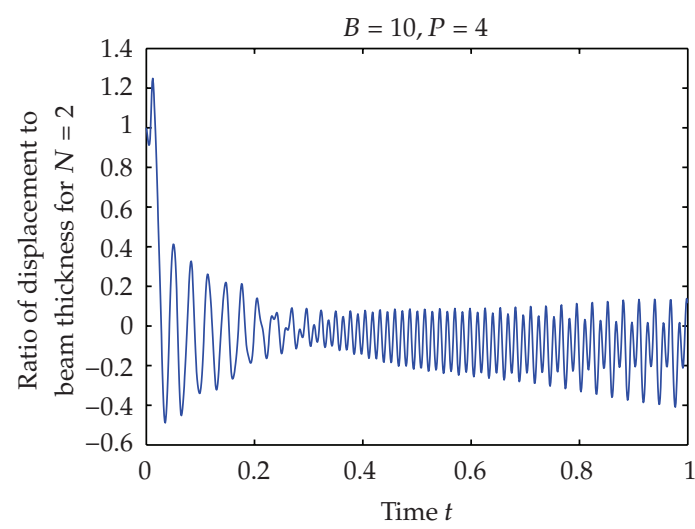

(b)

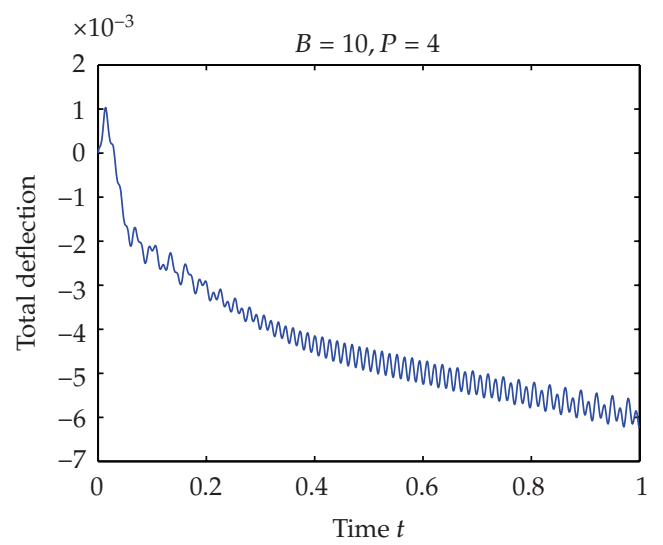

(d)

Figure 8: Divergent response for an ME beam with simply supported boundary conditions subjected to axial load and magnetic field outside the stable region.

the variables; as a result, the stable criteria for the magnetoelastic beam can be acquired after dissecting the nonlinear Schrodinger equation and requiring the imaginary part of the time domain solution to be vanished. Four kinds of boundary conditions are imposed to a low-carbon steel beam structure as examples in order to implement the proposed methodology, and the effects of both initial conditions and boundary conditions on the stability criterion of the ME beam are examined in detail. Stability criterion curve for the dispersion equation of the ME beam is firstly depicted in order to reveal the magnificent influence of the structure characteristic itself, followed by the instability constraint due to the variation of initial conditions and the observation locations. The prior one is actually denoting a parabola, whereas the latter one is sometimes a diamond-like or ellipse-like region spotting along the prior one. By the fact that the strong instability due to the initial conditions and observation points makes the analysis become more complicated, a slight change in the number of instability diamond can be noticed with respect to different boundary conditions, and an extra added instability ellipse centered in the vicinity of zero magnetic field is also detected for the cases other than simply supported beam. As we expected, the larger value of spring constant results in the wider stability region to the ME beam system, that is, spring foundation plays a role in stabilizing the system. 


\section{Acknowledgments}

This work was partially supported by the National Science Council of the Republic of China under Grant NSC 95-2115-M-214-002 and by I-Shou University of the Republic of China under Grant ISU 94-02-22. The authors are grateful for these supports.

\section{References}

[1] J. R. Reitz, F. J. Milford, and R. W. Christy, Foundations of Electromagnetic Theory, Wesley, New York, NY, USA, 1992.

[2] F. C. Moon, Magneto-Solid Mechanics, Wiley, New York, NY, USA, 1984.

[3] F. C. Moon and Y. H. Pao, "Magneto-elastic buckling of a thin plate," Journal of Applied Mechanics, vol. 37, pp. 53-58, 1968.

[4] D. V. Wallerstein and M. O. Peach, "Magneto-elastic buckling of beams and thin plates of magnetically soft material," Journal of Applied Mechanics, vol. 39, pp. 451-455, 1972.

[5] K. Miya, K. Hara, and K. Someya, "Experimental and theoretical study on magneto-elastic buckling of ferro-elastic cantilevered beam-plate," Journal of Applied Mechanics, vol. 45, no. 2, pp. 355-360, 1978.

[6] F. C. Moon and Y. H. Pao, "Vibration and dynamic instability of a beam-plate in a transverse magnetic field," Journal of Applied Mechanics, vol. 36, pp. 92-100, 1969.

[7] H. Kojima, K. Nagaya, H. Shiraishi, and A. Yamashita, "Nonlinear vibration of a beam with a mass subjected to alternating electromagnetic force," Bulletin of the JSME, vol. 28, no. 237, pp. 468-474, 1985.

[8] Y.-S. Shih, G.-Y. Wu, and E. J. S. Chen, "Transient vibrations of a simply-supported beam with axial loads and transverse magnetic fields," Mechanics of Structures and Machines, vol. 26, no. 2, pp. 115-130, 1998.

[9] M.-F. Liu and T.-P. Chang, "Vibration analysis of a magneto-elastic beam with general boundary conditions subjected to axial load and external force," Journal of Sound and Vibration, vol. 288, no. 1-2, pp. 399-411, 2005.

[10] G. Y. Wu, "The analysis of dynamic instability and vibration motions of a pinned beam with transverse magnetic fields and thermal loads," Journal of Sound and Vibration, vol. 284, no. 1-2, pp. 343-360, 2005.

[11] B. Pratiher and S. K. Dwivedy, "Parametric instability of a cantilever beam with magnetic field and periodic axial load," Journal of Sound and Vibration, vol. 305, no. 4-5, pp. 904-917, 2007.

[12] D. J. Hasanyan, G. M. Khachaturyan, and G. T. Piliposyan, "Mathematical modeling and investigation of nonlinear vibration of perfectly conductive plates in an inclined magnetic field," Thin-Walled Structures, vol. 39, no. 1, pp. 111-123, 2001.

[13] C. Bu, "Generalized solutions to the cubic Schrödinger equation," Nonlinear Analysis: Theory, Methods $\mathcal{E}$ Applications, vol. 27, no. 7, pp. 769-774, 1996. 


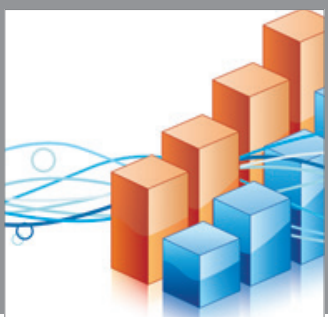

Advances in

Operations Research

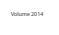

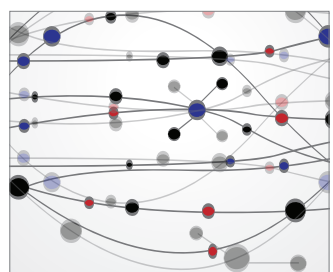

\section{The Scientific} World Journal
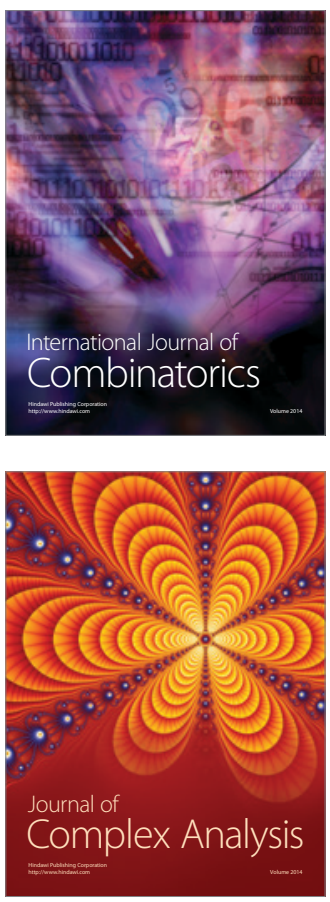

International Journal of

Mathematics and

Mathematical

Sciences
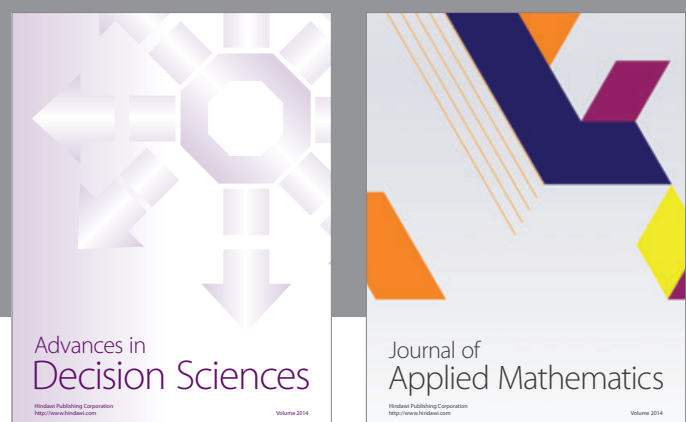

Journal of

Applied Mathematics
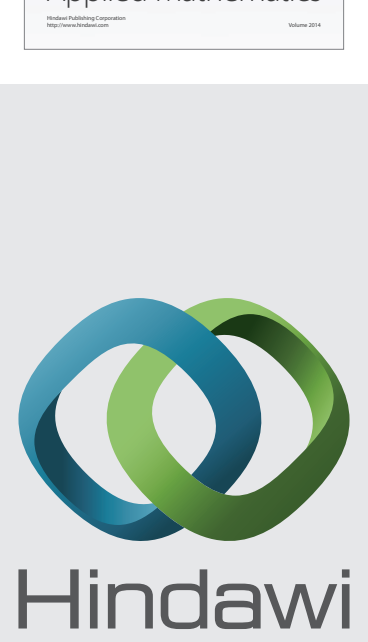

Submit your manuscripts at http://www.hindawi.com
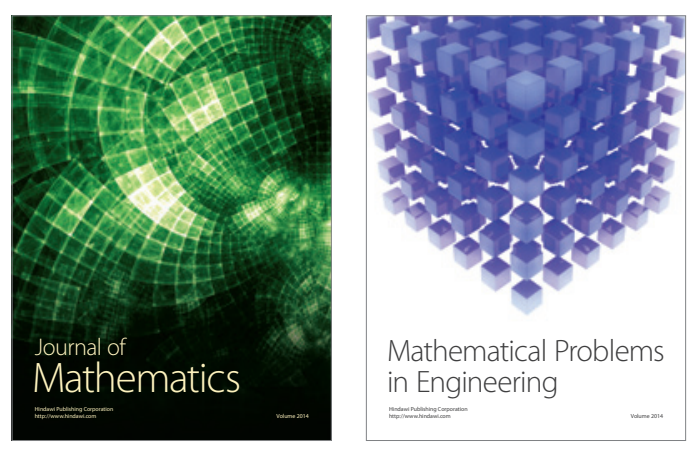

Mathematical Problems in Engineering
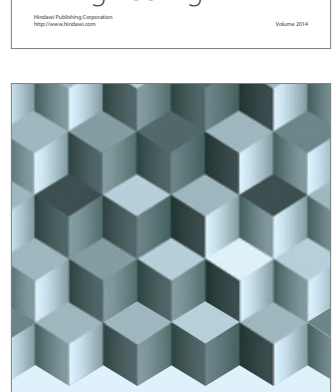

Journal of

Function Spaces
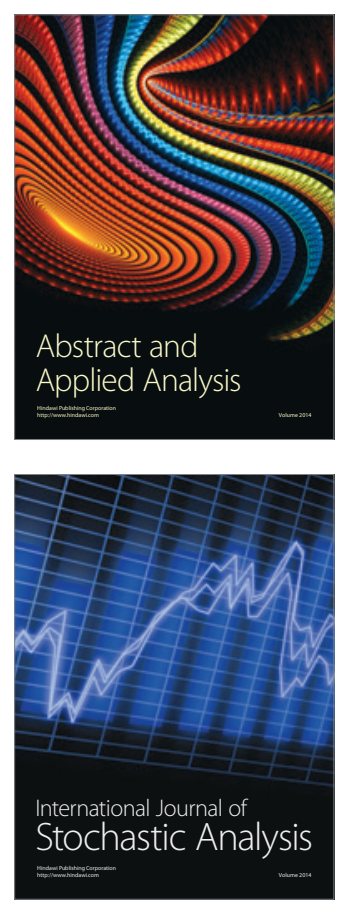

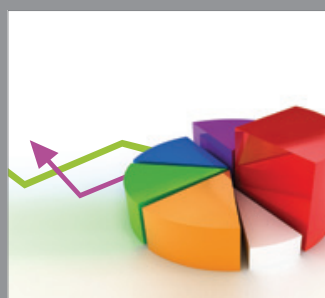

ournal of

Probability and Statistics

Promensencen
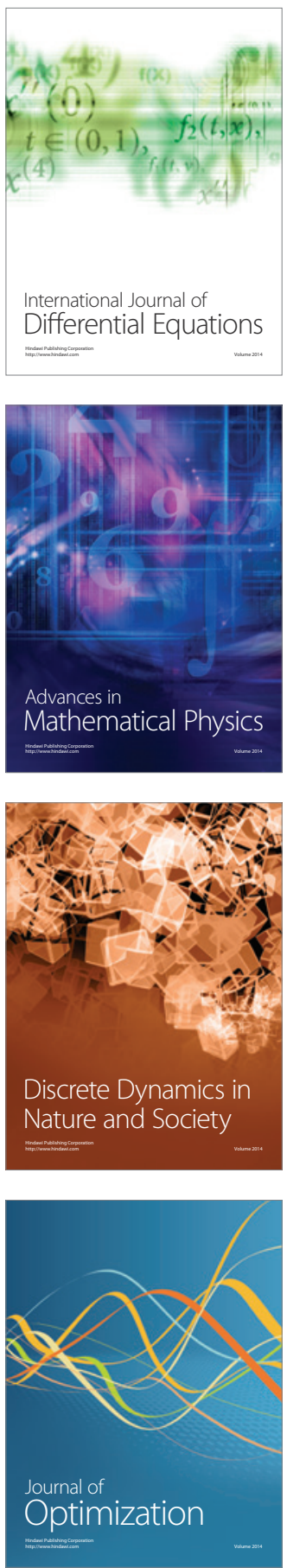